\title{
An Abstraction Technique for Producing 3D Visual Contents
}

\author{
Kyungha Min \\ Dept. of Digital Media, Sangmyung Univ., Seoul, Korea \\ minkyungha@gmail.com
}

\begin{abstract}
We present an image processing algorithm that abstracts video and digital animation to produce $3 D$ visual contents. Our algorithm prepares different projection matrices for both eyes and applies both matrices to produce the left image and right image for $3 D$ contents. We also preserve the coherence between the both images by comparing them and modifying one image to the other image. We test our scheme for video and digital animation and produce $3 D$ contents.
\end{abstract}

Keywords: image abstraction, 3D visual contents, video, digital animation, mean shift

\section{Introduction}

Abstraction, one of the important techniques in non-photorealistic rendering (NPR), is used in many applications for producing attractive and impressive visual contents [1-6]. In the early days, the abstract aims a stylization of color image that produces very impressive styled images [1]. This scheme is further extended to be able to be applied to a video with temporally coherent result [2]. Currently, the status of abstraction techniques preserves the details of images while it abstracts unnecessary parts [3-5]. Some pursue the artistic quality of stylization [6].

In the industry, the movie "Avatar" made 3D contents as a key trend in producing visual contents. Big studios in Hollywood are encouraged to put a large budget in producing new 3D movies and in converting the existing movies into 3D movies. The conversion process, however, takes a lot of time and experienced experts. For producing 3D animations using video abstraction technique, we present a video abstraction scheme that converts the video or 2D animation into 3D contents without user's intervention.

We present a video abstraction scheme for producing 3D contents. The input of our system is either stereoscopic video captured using stereoscopic camcorder or digital animation rendered using stereoscopic renderer. We apply a video abstraction algorithm to the both frames and produce 3D contents. The abstraction we apply is designed based on mean shift algorithm [7]. For an efficient abstraction, we extend the mean shift algorithm into a hierarchical structure that produces a multi-scale abstraction results. The coherence of the abstracted images is preserved by modifying one abstracted image into other. This process is illustrated in Figure 1.

This paper is organized as follows. In Section 2, we briefly review related work on abstraction and stereoscopic non-photorealistic rendering techniques. We present our video abstraction scheme in Section 3 and explain the scheme that preserves coherence in Section 4. In Section 5, we suggest the results. We conclude this paper and draw future directions in Section 6. 


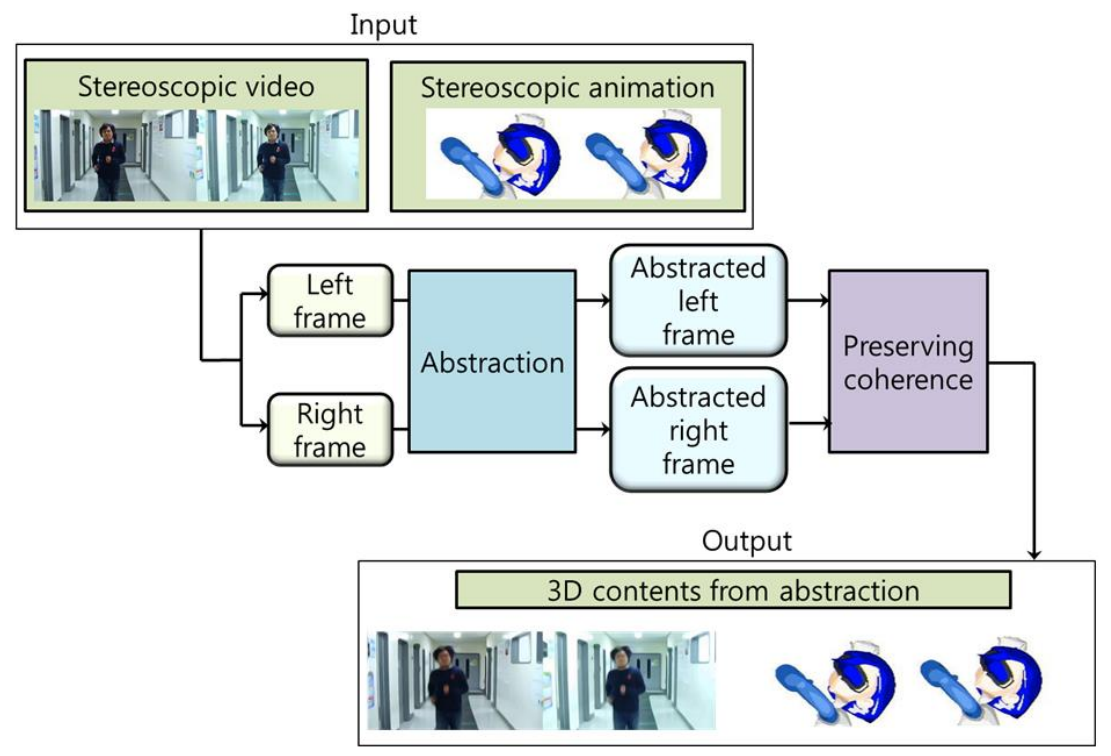

Figure 1. The overview of the algorithm

\section{Related work}

\subsection{Abstraction}

Image abstraction is a key technique in applying artistic effects to an image. DeCarlo et al. [1] presented a selective abstraction scheme for photographs, which controls the level of abstraction by tracking users' eye. They used mean shift operations to build their basic abstraction framework. Their scheme, however, does not preserve temporal coherence of their results, which prevents us to apply their scheme for video abstraction.

Winnenmoller et al., [2] presented DoG (difference of Gaussian) filter and median filter to abstract video in realtime. The color from median filter is quantized to finalize the abstraction. They also present an interesting user test on perception that the abstracted images are better remembered to users than the original photographs.

Kang et al., [3] developed a flow-based abstraction scheme that utilizes the edge tangent flow (ETF) computation and the line integral convolution (LIC). The image is abstracted by integrating the colors sampled along a smooth flow computed at each pixel. They [4] also present a very coherent line drawing algorithm using the ETF and LIC.

Kyprianidis and Kang [5] presented a coherence-enhancing stylization scheme for color images and videos. They achieved their coherence by adding the line integral algorithm with directional shock filter.

Gerstner et al., [6] presented an interesting abstraction scheme for color images whose results become similar to low-resolution pixels. They mapped the features of high resolution image into low resolution space and reduced color palette to construct the target image.

\subsection{Stereoscopic NPR}

Gelautz et al., proposed a scheme that automatically applies artistic effects on stereoscopic images and video [8]. They used depth maps of both images to preserve stereoscopic coherence and to control the artistic effects of the regions with different depths. Stavrakis and Gelautz developed a stereoscopic painter's algorithm [9], which renders stereoscopic images using a depth map-based painters' algorithm. 
Markovic et al., developed an automatic scheme that expresses sketch effects from a stereoscopic scene [10]. They extract edge combined image by estimating and comparing the depth maps of both images and execute a parametric curve fitting algorithm to the pixels on the extracted edges.

Stavrakis et al., executed a series of works on stereoscopic NPR in 2005 [11-13]. They proposed a stereoscopic stylization scheme that propagates the artistic effects expressed on one image to the other image incrementally [11]. This scheme is improved by applying a multi-scale control scheme to the stylization effects [12]. Furthermore, they extended the stereoscopic stylization scheme to stereoscopic painting scheme [13].

Markovic and Gelautz presented a cartoon drawing scheme on stereoscopic video [14]. They compute the depth maps from both images of each frame, and use them to extract stroke directions on the images. The result of their scheme preserves the perspectiveness of the original images. Starvrakis et al., presented an interactive system in stereoscopic NPR [15]. Using this system, users can express their desired effects such as drawing, stylization, and painting on stereoscopic images.

\section{Video Abstraction}

We apply a selective abstraction algorithm designed based on a mean-shift algorithm [7] to abstract the individual frames of video. The mean-shift algorithm simplifies an image by quantizing colors of pixels by finding the mean color of a region surrounding the pixels. We consider $h_{s}$ to determine the region of a pixel and $h_{r}$ to the range of colors.

The selective abstraction is achieved by abstracting the target image in various resolutions. From the finest resolution, we reduce the resolution by $1 /$ sqrt(2). This strategy constructs the hierarchy of the resolutions as illustrated in Figure 2. This hierarchy leads us to build an abstraction pyramid where the abstraction of the highest resolution exists at the bottom of the pyramid (See Figure 3). The overlap of the regions as illustrated in Figure 2 is sought by finding the overlap of the area. We estimate the overlapped region using the following

$$
\operatorname{overlap}\left(A, B_{i}\right)=\frac{\operatorname{area}\left(A \cap B_{i}\right)}{\left\|\operatorname{color}(A)-\operatorname{color}\left(B_{i}\right)\right\|+1}
$$

formula:

In the above formula, $A$ denotes the area belonging to the lower level of the tree and $B_{i}$ denotes the area lying in the higher level of $A$ 's level. We aim is to find $i$ that maximizes overlap $\left(A, B_{i}\right)$ for all $B_{i}$ 's. The result of stereoscopic abstraction is illustrated in Figure 4.

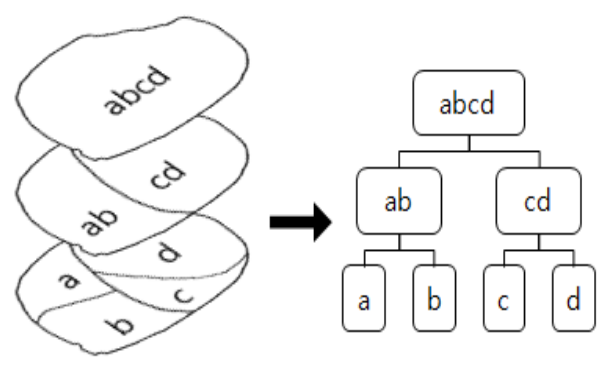

Figure 2. The hierarchical image abstraction and its corresponding tree structure 


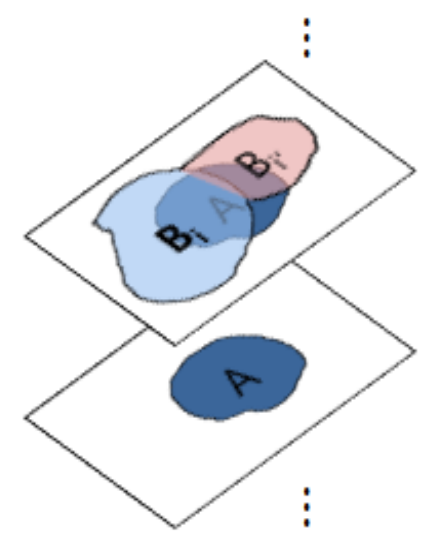

Figure 3. Overlapping of the abstracted area

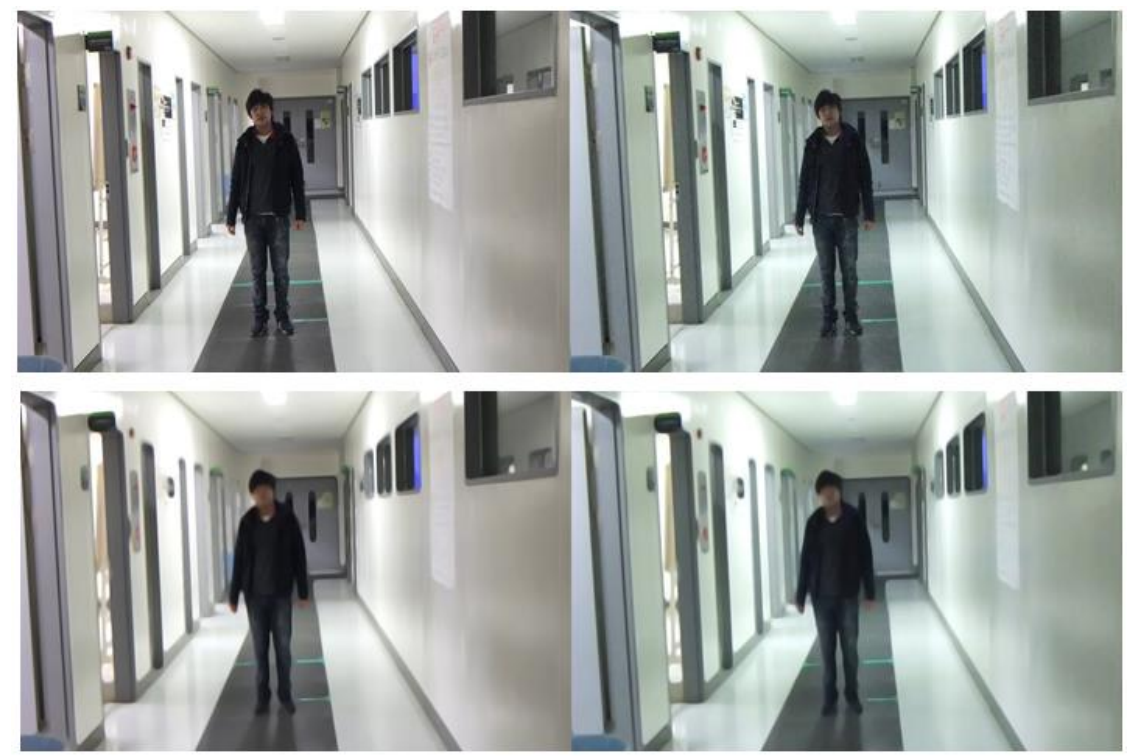

Figure 4. Result of stereoscopic abstraction

\section{Preserving Coherence}

We design an algorithm to preserve the coherence of the abstracted images. In the existing works, the coherence between the left image and the right image is preserved in two steps: (i) applying the artistic style in one image and (ii) applying the style to the corresponding point of the other image. These schemes have a problem in applying the images with occlusion or disocclusions where the objects in both image do not match. Our scheme compares the abstracted images and reduces the difference by deforming one image into other. We fix the right abstracted image and deform the left image, if user's right eye plays the role of major visual perception.

The abstracted left image is denoted as $I_{L}$ and the right image is as $I_{R}$. The $P_{L}$ and $P_{R}$ denote the projection matrix for the left camera and right camera, respectively. Then, we 
estimate the abstracted left image from the right image by applying the projection of left camera following the inverse projection of right camera. $\mathrm{I}_{\mathrm{R}}$, the estimated abstract right image, are computed as $I_{R}{ }^{\prime}=P_{R} P_{L}^{-1} I_{R}$.

\section{Implementation and Results}

We implemented our algorithm on a PC with Pentium i7 CPU and 4 GB main memory. The result of algorithm applied to a video captured using a stereoscopic camcorder is illustrated in Figure 5. In this example, a subject is approaching from the end of the hall. The approach of the subject emphasizes the stereoscopic feeling of the results. The abstraction of this video takes $2.4 \mathrm{sec} /$ frames in average. In Figure 6, we suggest a stereoscopic digital animation. These animations are rendered in 3-4 frames per second.

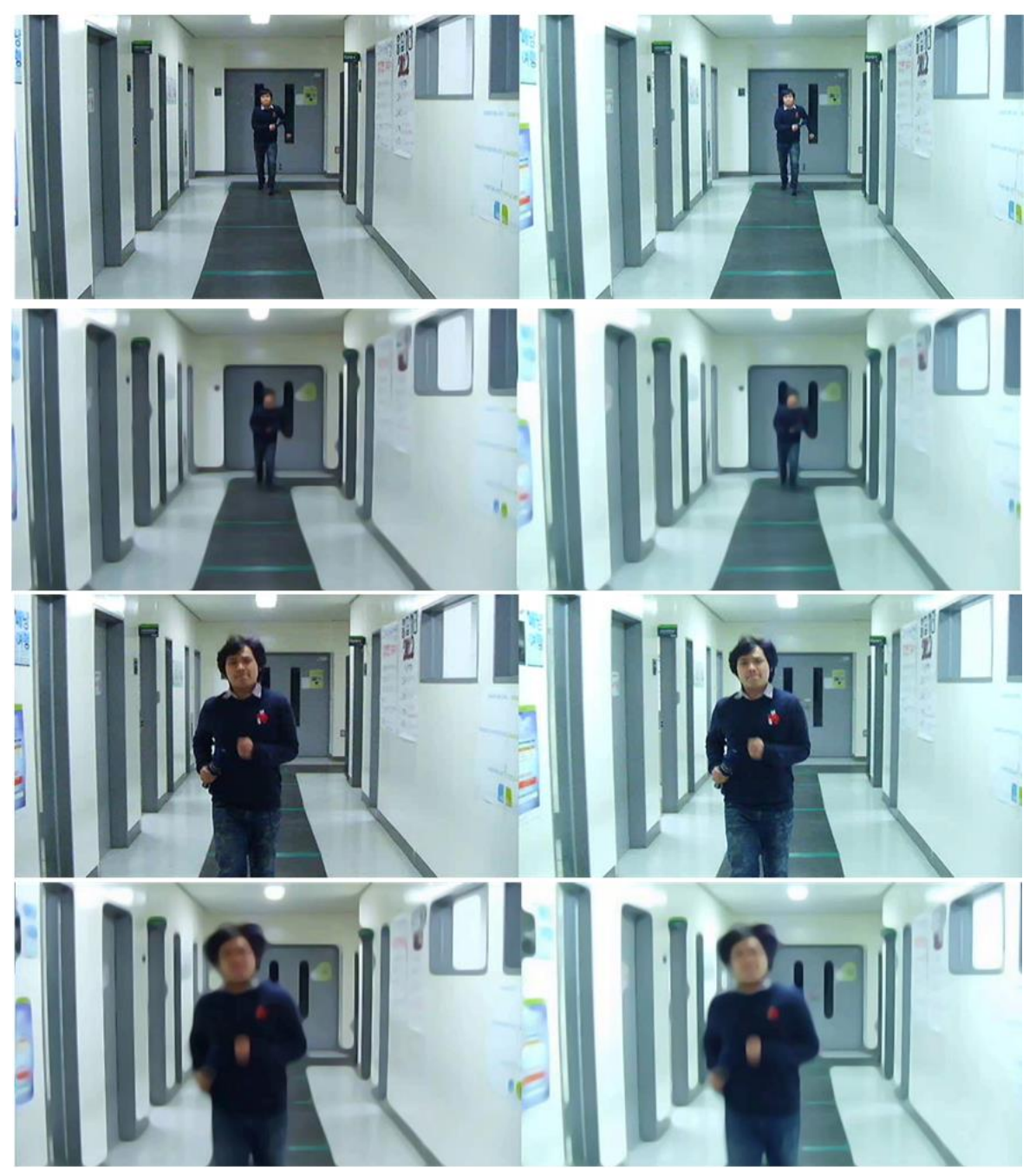

Figure 5. Result for a video 
International Journal of Multimedia and Ubiquitous Engineering Vol.8, No.5 (2013)
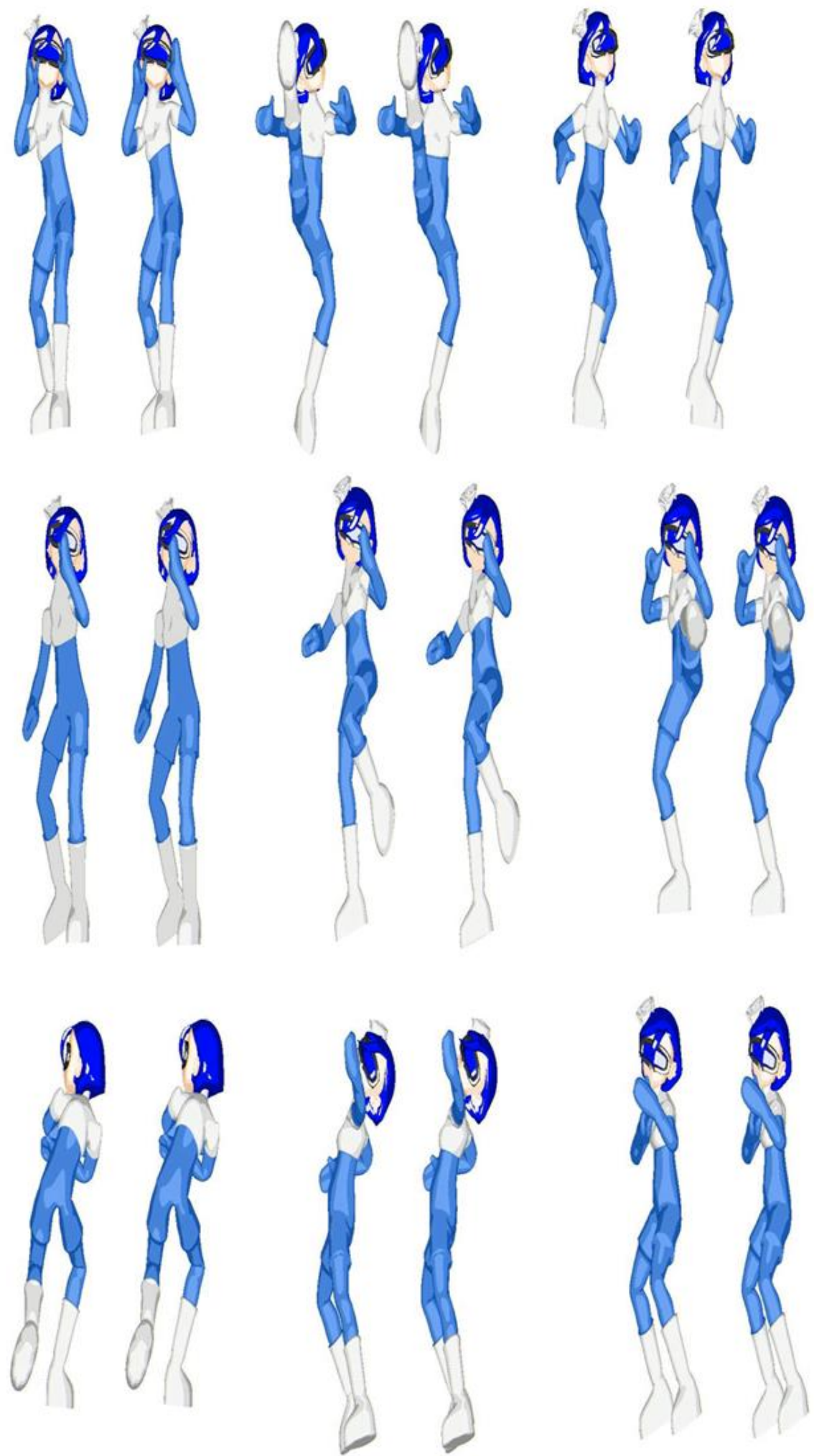

Figure 6. Result for a digital animation 


\section{Conclusion and Future Work}

We have presented a video abstraction scheme for producing 3D contents. Our algorithm abstracts the left frame and right frame of a video clip and preserves the temporal coherence of the abstracted frames.

We will extend our scheme to other NPR effects such as contouring and artistic media simulation and produce $3 \mathrm{D}$ contents.

\section{Acknowledgements}

This research was supported by a 2013 Research Grant from Sangmyung University.

\section{References}

[1] D. Decarlo and A. Santella, "Stylization and Abstraction of Photographs", Proceedings of SIGGRAPH 2002, (2002), pp. 769-776.

[2] H. Winnemoller, S. Olsen and B. Gooch, "Real-Time Video Abstraction", Proceedings of SIGGRAPH 2006, (2006), pp. 1221-1226.

[3] H. Kang, S. Lee and C. Chui, "Flow-Based Image Abstraction", IEEE Trans. on Visualization and Computer Graphics, vol. 15, no. 1, (2009), pp. 62-76.

[4] H. Kang, S. Lee and C. Chui, "Coherent Line Drawing”, In NPAR, (2007), pp. 43-50.

[5] J. E. Kyprianidis and H. Kang, "Image and Video Abstraction by Coherence-Enhancing Filtering", Computer Graphics Forum, vol. 30, no. 2, (2011), pp. 593-602.

[6] T. Gerstner, D. DeCarlo, M. Alexa, A. Finkelstein, Y. Gingold and A. Nealen, "Pixelated Image Abstraction", In NPAR 2012, (2012), pp. 71-78.

[7] D. Comaniciu and P. Meer, "Mean shift: A robust app.roach toward feature space analysis", IEEE Trans. on Pattern Analysis and Machine Intelligence, vol. 24, no. 5, (2002), pp. 603-619.

[8] M. Gelautz, E. Stavrakis and M. Bleyer, "Stereo-Based Image and Video Analysis for Multimedia Applications", Int. Arch. of Photogrammetry, Remote Sensing and Spatial Information Sciences, vol. 35, (2004), pp. 998-1003.

[9] E. Stavrakis and M. Gelautz, "Image-based stereoscopic painterly rendering", Proc. of EGSR 2004, (2004), pp. 53-60.

[10] D. Markovic, E. Stavrakis and M. Gelautz, "Parameterized Sketches from Stereo Images", Proc. of the 17th Annual Symposium on Electronic Imaging: Science and Technology, vol. 5685, (2005), pp. 783-791.

[11] E. Stavrakis, M. Bleyer, D. Markovic and M. Gelautz, "Image-based stereoscopic stylization", Proc. of IEEE International Conference on Image Processing (ICIP) 2005, (2005), pp. 5-8.

[12] E. Stavrakis and M. Gelautz, "Stereoscopic painting with varying levels of detail", Proc. of SPIEStereoscopic displays and virtual reality systems XII, vol. 5664, (2005), pp. 250-259.

[13] E. Stavrakis and M. Gelautz, "Computer Generated Stereoscopic Artwork", Proc. of the First Eurographics Workshop on Computational Aesthetics in Graphics, Visualization and Imaging 2005 (CAe'05), (2005), pp. 143-149.

[14] D. Markovic and M. Gelautz, "Comics-Like Motion Depiction from Stereo", Proc. of the 14-th International Conference in Central Europe on Computer Graphics, Visualization and Computer Vision (WSCG '06), (2006), pp. 155-160.

[15] E. Stavrakis and M. Gelautz, "Interactive Tools for Image-Based Stereoscopic Artwork", Proc. of SPIE Stereoscopic Displays and Applications XIX, vol. 6803, SPIE, (2008). 
International Journal of Multimedia and Ubiquitous Engineering Vol.8, No.5 (2013)

\section{Author}

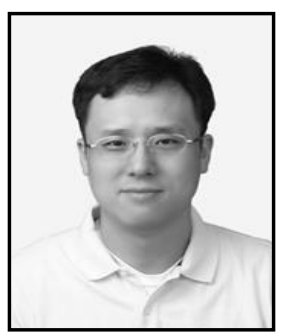

\section{Kyungha Min}

Kyungha Min got his Ph.D at POSTECH at 2000. His research interest is computer graphics and image processing. Currently he is at Dept. of Digital Media, Sangmyung Univ., Seoul, Korea. 